\title{
Molecules in metastasis
}

\author{
Jonathan P. Sleeman ${ }^{1,2}$
}

Published online: 18 March 2019

(c) Springer Nature B.V. 2019

In this issue, the first article in a new manuscript category Molecules in Metastasis is published. The aim of Molecules in Metastasis articles is to provide readers with an easily readable and pithy overview about the functional role of a single molecule or small family of molecules in the process of metastasis. These minireviews summarize the metastasis-relevant molecular biology and cellular functions of the molecule(s) concerned, outline their clinical relevance, and describe the possible therapeutic or translational potential of targeting the molecule(s) in question. Our intention is that thereby, newcomers to the metastasis research field will be able to get up to speed quickly with the current state of the art and understanding about the role key molecules play in metastasis.

The first article in the Molecules in Metastasis series focuses on ALCAM/CD166, a cell adhesion molecule that makes an important contribution to metastatic progression [1]. Lersner, Droesen and Zijlstra expertly introduce the reader to the regulation of ALCAM gene expression and the importance of alternative splicing for its function, turnover of the molecule through proteolysis and shedding, as well as key partners that ALCAM/CD166 interacts with. Potential therapeutic approaches, for example through interfering with the interaction between ALCAM and CD6 are also discussed.

The Editors of Clinical \& Experimental Metastasis are excited about the potential of the new Molecules in Metastasis article category, and are very pleased that the series has kicked off with such an excellent review. Please consider producing a Molecules in Metastasis article yourself about your own favorite metastasis-relevant molecule, and educate the community about its importance in the process of metastatic dissemination and secondary tumor formation.

Jonathan Sleeman Editor-in-Chief

\section{Reference}

1. Von Lersner A, Droesen D, Zijlstra A (2019) Modulation of cell adhesion and migration through regulation of the immunoglobulin superfamily member ALCAM/CD166. Clin Exp Metastasis. https ://doi.org/10.1007/s10585-019-09957-2

Publisher's Note Springer Nature remains neutral with regard to jurisdictional claims in published maps and institutional affiliations.

Jonathan P. Sleeman

sleeman@medma.uni-heidelberg.de

1 European Centre for Angioscience (ECAS), Medical Faculty Mannheim, University of Heidelberg, TRIDOMUS-Gebäude Haus C, Ludolf-Krehl-Str. 13 - 17, 68167 Mannheim, Germany

2 Karlsruhe Institute for Technology (KIT), Campus Nord, Institut für Toxikologie und Genetik, Postfach 3640, 76021 Karlsruhe, Germany 